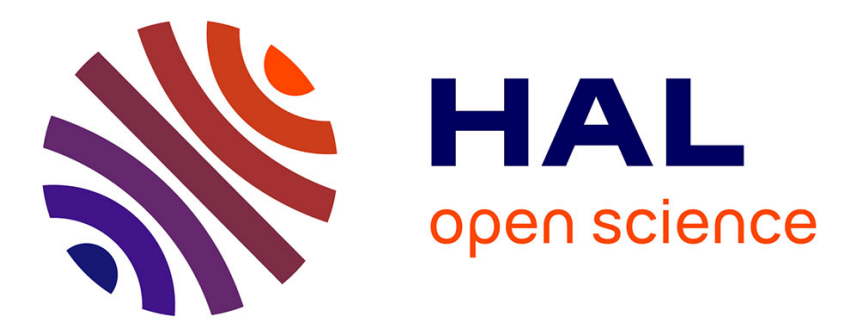

\title{
Implicitization of Bihomogeneous Parametrizations of Algebraic Surfaces via Linear Syzygies
}

Laurent Busé, Marc Dohm

\section{To cite this version:}

Laurent Busé, Marc Dohm. Implicitization of Bihomogeneous Parametrizations of Algebraic Surfaces via Linear Syzygies. International Conference on Symbolic and Algebraic Computation (ISSAC), Jul 2007, Waterloo, Ontario, Canada. pp.69-76, 10.1145/1277548.1277559 . inria-00168956

\section{HAL Id: inria-00168956 \\ https://hal.inria.fr/inria-00168956}

Submitted on 30 Aug 2007

HAL is a multi-disciplinary open access archive for the deposit and dissemination of scientific research documents, whether they are published or not. The documents may come from teaching and research institutions in France or abroad, or from public or private research centers.
L'archive ouverte pluridisciplinaire HAL, est destinée au dépôt et à la diffusion de documents scientifiques de niveau recherche, publiés ou non, émanant des établissements d'enseignement et de recherche français ou étrangers, des laboratoires publics ou privés. 


\title{
Implicitization of Bihomogeneous Parametrizations of Algebraic Surfaces via Linear Syzygies
}

\author{
Laurent Busé, Marc Dohm
}

August 30, 2007

\begin{abstract}
We show that the implicit equation of a surface in 3-dimensional projective space parametrized by bi-homogeneous polynomials of bi-degree $(d, d)$, for a given integer $d \geq 1$, can be represented and computed from the linear syzygies of its parametrization if the base points are isolated and form locally a complete intersection.
\end{abstract}

\section{Introduction}

Implicitization, i.e. finding the implicit equation of an algebraic curve or surface defined parametrically, is a classical problem and there are numerous approaches to its solution, most of them based either on resultants, Gröbner bases, or syzygies. A good historical overview of methods based on resultants or Gröbner bases can be found in [13] and [7].

Syzygy-based methods - also known as "moving surfaces" methods - were introduced in 13] and have been further developed in a number of publications. In the case of curves, these methods show that the linear syzygies of a given parametrization can be put together in a square matrix whose determinant is an implicit equation. Several attempts to generalize these results to the case of surfaces have been made. The construction of a square matrix whose determinant is the implicit equation requires the use of quadratic syzygies in addition to the linear syzygies. Moreover, these methods are only valid for birational parametrizations and need additional assumptions in the presence of base points.

Copyright ACM, 2007. This is the author's version of the work. It is posted here by permission of ACM for your personal use. Not for redistribution. The definitive version was published in Proceedings of the 2007 international Symposium on Symbolic and Algebraic Computation (Waterloo, Ontario, Canada, July 29 - August 01, 2007), ACM Press, New York, NY, pages 69-76, http://doi.acm.org/10.1145/1277548.1277559. 
Just to name some recent publications on syzygy-based methods, we cite [6], which treats the implicitization of base-point-free homogeneous parametrizations and [4], which does the same for parametrizations with base points. In [1] a determinantal representation of the implicit equation of a bi-homogeneous parametrization is constructed with linear and quadratic relations, whereas [12] gives such a construction in the toric case.

Recently it has been proved in [5] and [3] that surfaces parametrized by the projective plane can be represented and computed only using the linear syzygies of the parametrization, in the case where the base points are isolated and locally complete intersections. In some sense, this result is a natural generalization of the method of "moving curves" developed for planar curves in [13]; the only difference is that the matrix obtained in the case of surfaces is not square, but still represents the surface (see the end of Section 3 for a detailed explanation of this term).

In this paper our main objective is to develop a similar implicitization technique for surfaces given by bi-homogeneous parametrizations, which are of interest for a number of applications in geometric modelling and computer-aided design. We will show that also in this case the surface can be represented by a non-square matrix constructed by only using linear syzygies and we will explain how to efficiently compute this matrix with standard computer algebra systems.

More precisely, we focus on the following problem. Let $\mathbb{K}$ be any field (all the varieties we will consider hereafter are understood to be taken over $\mathbb{K}$ ). We suppose given a rational map

$$
\begin{aligned}
\mathbb{P}^{1} \times \mathbb{P}^{1} & \stackrel{\phi}{\rightarrow} \mathbb{P}^{3} \\
(s: u) \times(t: v) & \mapsto\left(f_{1}: f_{2}: f_{3}: f_{4}\right)(s, u, t, v)
\end{aligned}
$$

where each polynomial $f_{1}, f_{2}, f_{3}, f_{4}$ is bi-homogeneous of bi-degree $(d, d), d$ being a given positive integer, with respect to the homogeneous variables $(s: u)$ and $(t: v)$. We assume that

- $\phi$ parametrizes a surface $\mathscr{H}$ (which is equivalent to require that $\phi$ is a generically finite map onto its image) which is hence irreducible

- the greatest common divisor of $f_{1}, f_{2}, f_{3}, f_{4}$ is a non-zero constant which essentially requires the number of base points of $\phi$ to be finite (possibly zero).

We aim to find a representation of $\mathscr{H}$ in terms of linear syzygies of $f_{1}, f_{2}, f_{3}$ and $f_{4}$ similar to the known ones for plane curves and for space surfaces parametrized by the projective plane.

The paper is organized as follows. In Section 2 we give an equivalent formulation of our problem which replaces the given $\mathbb{N} \times \mathbb{N}$-graduation by a single $\mathbb{N}$-graduation. In Section 3 we will introduce an associated approximation complex that will be used in Section 3.3 to prove our main result. Then an algorithmic version is detailed in Section 4, as well as an illustrative example. 


\section{The Segre embedding}

It is well-known that $\mathbb{P}^{1} \times \mathbb{P}^{1}$ can be embedded in $\mathbb{P}^{3}$ through the so-called Segre embedding

$$
\begin{aligned}
\mathbb{P}^{1} \times \mathbb{P}^{1} & \stackrel{\rho}{\rightarrow} \mathbb{P}^{3} \\
(s: u) \times(t: v) & \mapsto(s t: s v: u t: u v) .
\end{aligned}
$$

We denote by $\mathscr{S}$ its image, which is an irreducible surface of degree 2 in $\mathbb{P}^{3}$, whose equation in the coordinates $X_{1}, X_{2}, X_{3}, X_{4}$ of $\mathbb{P}^{3}$ is known to be $X_{1} X_{4}-$ $X_{2} X_{3}$. Our strategy to solve our implicitization problem is to reparametrize the surface $\mathscr{H}$ by $\mathscr{S} \subset \mathbb{P}^{3}$, that is to say to consider $\mathscr{H}$ as the closed image of the map $\psi$ from $\mathscr{S}$ to $\mathbb{P}^{3}$ fitting in the commutative diagram

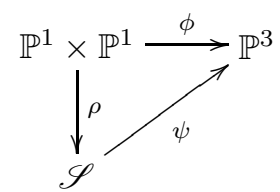

In the rest of this paper we will use the map $\psi=\phi \circ \rho^{-1}$ to implicitize $\mathscr{H}$, which has the advantage of replacing the $\mathbb{N} \times \mathbb{N}$-graduation of $\mathbb{P}^{1} \times \mathbb{P}^{1}$ by a single $\mathbb{N}$-graduation. In order to justify this approach we need to describe explicitly the algebraic counterparts of the maps in the above diagram.

We begin with the map $\phi$. The polynomial ring $\mathbb{K}[s, u]$ is canonically $\mathbb{N}$ graded,

$$
\mathbb{K}[s, u]=\bigoplus_{n \in \mathbb{N}} \mathbb{K}[s, u]_{n}=\mathbb{K}[s, u]_{0} \oplus \mathbb{K}[s, u]_{1} \oplus \mathbb{K}[s, u]_{2} \oplus \ldots
$$

where $\mathbb{K}[s, u]_{i}$ denotes the degree $i$ homogeneous component of $\mathbb{K}[s, u]$, and its homogeneous spectrum is the projective line, i.e. $\operatorname{Proj}(\mathbb{K}[s, u])=\mathbb{P}_{\mathbb{K}}^{1}$. Of course, the same is true for the polynomial ring $\mathbb{K}[t, v]$. Now, consider the $\mathbb{N}$-graded $\mathbb{K}$-algebra

$$
S:=\bigoplus_{n \in \mathbb{N}}\left(\mathbb{K}[s, u]_{n} \otimes_{\mathbb{K}} \mathbb{K}[t, v]_{n}\right) \subset \mathbb{K}[s, u] \otimes_{\mathbb{K}} \mathbb{K}[t, v]
$$

which is finitely generated by $S_{1}$ as an $S_{0}$-algebra. Then $\mathbb{P}^{1} \times \mathbb{P}^{1}$ is the homogeneous spectrum $\operatorname{Proj}(S)$ of $S$. Introducing new indeterminates $T_{1}, T_{2}, T_{3}, T_{4}$, the map $\phi$ is hence induced by the graded $k$-algebra morphism

$$
\begin{aligned}
\mathbb{K}\left[T_{1}, T_{2}, T_{3}, T_{4}\right] & \stackrel{p}{\rightarrow} S \\
T_{i} & \mapsto f_{i}(s, u, t, v) \quad i=1, \ldots, 4 .
\end{aligned}
$$

By [5, Theorem 2.1], $\operatorname{ker}(p) \subset \mathbb{K}\left[T_{1}, T_{2}, T_{3}, T_{4}\right]$ is the defining ideal of the closed image of $\phi$ in $\mathbb{P}^{3}=\operatorname{Proj}\left(\mathbb{K}\left[T_{1}, \ldots, T_{4}\right]\right)$; it is prime (since $S$ is a domain) and principal (since it is of codimension one by hypothesis and $\mathbb{K}\left[T_{1}, T_{2}, T_{3}, T_{4}\right]$ is factorial), i.e. any generator of $\operatorname{ker}(p)$ gives an equation of $\mathscr{H}$. 
We now turn to the Segre embedding $\rho$. As we did for the map $\phi$ (note that the Segre embedding is itself a parametrization of a surface in projective space) the map $\rho$ is induced by the graded $k$-algebra morphism

$$
\begin{aligned}
\mathbb{K}\left[X_{1}, X_{2}, X_{3}, X_{4}\right] & \stackrel{\theta}{\rightarrow} S \\
X_{1} & \mapsto s t \\
X_{2} & \mapsto s v \\
X_{3} & \mapsto u t \\
X_{4} & \mapsto u v .
\end{aligned}
$$

However, in this case $\theta$ is surjective and graded (it preserves the degree). Moreover, it is easy to see ${ }^{1}$ that its kernel is the principal ideal $\left(X_{1} X_{4}-X_{2} X_{3}\right) \subset$ $\mathbb{K}\left[X_{1}, X_{2}, X_{3}, X_{4}\right]$. Therefore, $\theta$ induces a graded isomorphism of $\mathbb{N}$-graded $\mathbb{K}$ algebras

$$
\bar{\theta}: A:=\mathbb{K}\left[X_{1}, X_{2}, X_{3}, X_{4}\right] /\left(X_{1} X_{4}-X_{2} X_{3}\right) \stackrel{\sim}{\longrightarrow} S
$$

which identifies $\mathbb{P}^{1} \times \mathbb{P}^{1}=\operatorname{Proj}(S)$ with the Segre variety $\mathscr{S}=\operatorname{Proj}(A) \subset \mathbb{P}^{3}=$ $\operatorname{Proj}\left(\mathbb{K}\left[X_{1}, X_{2}, X_{3}, X_{4}\right]\right)$.

We are now ready to describe $\psi$. This map is of the form

$$
\begin{aligned}
\mathscr{S} \subset \mathbb{P}^{3} & \stackrel{\psi}{\rightarrow} \mathbb{P}^{3} \\
\left(X_{1}: X_{2}: X_{3}: X_{4}\right) & \mapsto\left(g_{1}: g_{2}: g_{3}: g_{4}\right)\left(X_{1}, X_{2}, X_{3}, X_{4}\right)
\end{aligned}
$$

where $g_{1}, g_{2}, g_{3}, g_{4}$ are homogeneous polynomials of the same degree in $\mathbb{K}\left[X_{1}, X_{2}, X_{3}, X_{4}\right]$. By the graded isomorphism $\bar{\theta}$, it follows that $\operatorname{deg}(\phi)=$ $\operatorname{deg}(\psi)$ (we understand co-restriction to $\mathscr{H}$ ) and also that the $g_{i}$ 's must have degree $d$. To give an algorithmic construction we just have to determine the inverse map of $\bar{\theta}$. To do this, for all $n \in \mathbb{N}$ define the integer $k_{i, j}^{(n)}:=\max (0, n-$ $i-j$ ) and consider the map

$$
\begin{aligned}
S_{n} & \stackrel{\omega_{n}}{\longrightarrow} \mathbb{K}\left[X_{1}, X_{2}, X_{3}, X_{4}\right]_{n} \\
s^{i} u^{n-i} t^{j} v^{n-j} & \mapsto X_{1}^{i+j-n+k_{i, j}^{(n)}} X_{2}^{n-j-k_{i, j}^{(n)}} X_{3}^{n-i-k_{i, j}^{(n)}} X_{4}^{k_{i, j}^{(n)}}
\end{aligned}
$$

(for all couples $(i, j) \in\{0, \ldots, n\}^{2}$ ). Then, we define the map

$$
\omega:=\bigoplus_{n \in \mathbb{N}} \omega_{n}: S \rightarrow \mathbb{K}\left[X_{1}, X_{2}, X_{3}, X_{4}\right]
$$

which induces the inverse of $\bar{\theta}$ by passing to $A=\mathbb{K}\left[X_{1}, X_{2}, X_{3}, X_{4}\right] /\left(X_{1} X_{4}-\right.$ $X_{2} X_{3}$ ) (this is easy to check). Observe also that no monomial in the image of

\footnotetext{
${ }^{1}$ We clearly have $\left(X_{1} X_{4}-X_{2} X_{3}\right) \subset \operatorname{ker}(\theta)$. Now, if $P \in \operatorname{ker}(\theta)$ we deduce by a pseudoeuclidean division that there exists $N \in \mathbb{N}^{\star}$ such that

$$
X_{4}^{N} P=Q\left(X_{1}, \ldots, X_{4}\right)\left(X_{1} X_{4}-X_{2} X_{3}\right)+R\left(X_{2}, X_{3}, X_{4}\right) .
$$

But then $R \in \operatorname{ker}(\theta)$ and it is obvious to check that we have $\mathbb{K}\left[X_{2}, X_{3}, X_{4}\right] \cap \operatorname{ker}(\theta)=0$.
} 
$\omega$ is divisible by $X_{1} X_{4}$, so our representation of the inverse of $\bar{\theta}$ can be thought of as already reduced. Moreover, the coefficients of the $f_{i}$ 's and the $g_{i}$ 's are in correspondence: only the monomials are changed by $\omega$.

Therefore, we proved

Proposition 1 Defining for all $i=1,2,3,4$ the homogeneous polynomial

$$
g_{i}\left(X_{1}, X_{2}, X_{3}, X_{4}\right):=\omega\left(f_{i}(s, u, t, v)\right) \in \mathbb{K}\left[X_{1}, X_{2}, X_{3}, X_{4}\right]_{d},
$$

the map (2) is a parametrization of the surface $\mathscr{H} \subset \mathbb{P}^{3}$ with the property that $\operatorname{deg}(\psi)=\operatorname{deg}(\phi)$.

Furthermore, we actually proved that our initial problem, namely the implicitization of $\phi$ in terms of syzygies, is equivalent to the same problem with the parametrization $\psi$ which is induced by the map

$$
\begin{aligned}
\mathbb{K}\left[T_{1}, T_{2}, T_{3}, T_{4}\right] & \stackrel{h}{\rightarrow} A \\
T_{i} & \mapsto g_{i}\left(X_{1}, X_{2}, X_{3}, X_{4}\right) .
\end{aligned}
$$

This can be summarized by the following commutative diagram, which is the algebraic translation of the diagram (1).

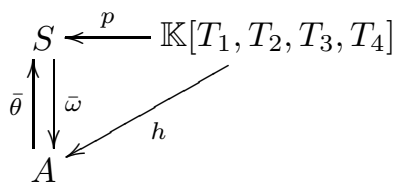

This shows that the syzygies of the $f_{i}$ 's over $S$ are in correspondence with the syzygies of the $g_{i}$ 's over $A$, in particular $k e r(h)=k e r(p)$. Moreover, it also shows that the base points of the parametrization $\phi$ are in one-to-one correspondence with the base points of the parametrization $\psi$ and that their local structure (complete intersection, multiplicity, etc.) is preserved by this correspondence.

Another interesting remark is the following: By [5, Theorem 2.5], we deduce that we have the equality

$$
\operatorname{deg}(\psi) \operatorname{deg}(\mathscr{H})=\operatorname{deg}(\mathscr{S}) d^{2}-\sum_{\mathfrak{p} \in V\left(g_{1}, \ldots, g_{4}\right) \cap \mathscr{S} \subset \mathbb{P}^{3}} e_{\mathfrak{p}}
$$

where $e_{\mathfrak{p}}$ denotes the algebraic multiplicity (in the sense of Hilbert-Samuel). Since it is immediate to check that $\operatorname{deg}(\mathscr{S})=2$ we recover the well-known formula of intersection theory (see [9, Prop. 4.4] or [7, Appendix]):

$$
\operatorname{deg}(\phi) \operatorname{deg}(\mathscr{H})=2 d^{2}-\sum_{\mathfrak{p} \in V\left(f_{1}, \ldots, f_{4}\right) \subset \mathbb{P}^{1} \times \mathbb{P}^{1}} e_{\mathfrak{p}} .
$$

Therefore, in the rest of this paper we will focus on the implicitization of $\psi$ by means of linear syzygies, which is a completely equivalent problem to our initial one. 


\section{The approximation complex}

For simplicity, we will denote by $X_{i}$ the classes of each variable in the quotient $\operatorname{ring} A=\mathbb{K}[\underline{X}] /\left(X_{1} X_{4}-X_{2} X_{3}\right)$, where $\underline{X}$ stands for the sequence $X_{1}, X_{2}, X_{3}, X_{4}$. Recall that $A$ is canonically graded, each variable having weight 1 . Let $I=$ $\left(g_{1}, g_{2}, g_{3}, g_{4}\right) \subset A$ be the ideal generated by the $g_{i}$ 's. We give a brief definition of the approximation complex of cycles associated to the sequence $g_{1}, g_{2}, g_{3}, g_{4}$ over $A$. This has been studied in depth in [11], see also [14]. Under certain conditions this complex is a free resolution of the symmetric algebra $\operatorname{Sym}_{A}(I)$, which is one of the main motivations for its study. Another essential feature of this complex is that - unlike the Koszul complex - its homology depends only on the ideal $\left(g_{1}, \ldots, g_{4}\right)$, not on the generators $g_{i}$. Here is the construction:

We consider the Koszul complex $\left(K \bullet(g, A), d_{\bullet}\right)$ associated to $g_{1}, \ldots, g_{4}$ over $A$ and denote $Z_{i}=\operatorname{ker}\left(d_{i}\right)$ and $B_{i}=\operatorname{im}\left(\overline{d_{i+1}}\right)$. It is of the form

$$
A(-4 d) \stackrel{d_{4}}{\rightarrow} A(-3 d)^{4} \stackrel{d_{3}}{\rightarrow} A(-2 d)^{6} \stackrel{d_{2}}{\rightarrow} A(-d)^{4} \stackrel{d_{1}}{\rightarrow} A
$$

where the differentials are matrices whose non-zero entries are $\pm g_{1}, \ldots, \pm g_{4}$. We introduce new variables $T_{1}, \ldots, T_{4}$ and set $\mathcal{Z}_{i}=Z_{i}(i \cdot d) \otimes_{A} A[\underline{T}]$, which we will consider as bi-graded $A[\underline{T}]$-modules (one grading is induced by the grading of $A$, the other one comes from setting $\operatorname{deg}\left(T_{i}\right)=1$ for all $i$ ). Now the approximation complex of cycles $\left(\mathcal{Z}_{\bullet}(\underline{g}, A), e_{\bullet}\right)$, or simply $\mathcal{Z}_{\bullet}$, is the complex

$$
0 \rightarrow \mathcal{Z}_{3}(-3) \stackrel{e_{3}}{\rightarrow} \mathcal{Z}_{2}(-2) \stackrel{e_{2}}{\rightarrow} \mathcal{Z}_{1}(-1) \stackrel{e_{1}}{\rightarrow} \mathcal{Z}_{0}
$$

where the differentials $e_{\bullet}$ are obtained by replacing $g_{i}$ by $T_{i}$ for all $i$ in the matrices of $d_{\bullet}$ (note that $\mathcal{Z}_{4}=0$, since $d_{4}$ is injective). It is an important remark that

$$
\begin{aligned}
\operatorname{im}\left(e_{1}\right) & =\left\{\sum_{i=1}^{4} p_{i} T_{i} \mid p_{i} \in A[\underline{T}], \sum_{i=1}^{4} p_{i} g_{i}=0\right\} \\
& =\left(\sum_{i=1}^{4} p_{i} T_{i} \mid p_{i} \in A, \sum_{i=1}^{4} p_{i} g_{i}=0\right) \subset A[\underline{T}]
\end{aligned}
$$

and therefore $H_{0}\left(\mathcal{Z}_{\bullet}\right)=A[\underline{T}] / \operatorname{im}\left(e_{1}\right) \simeq \operatorname{Sym}_{A}(I)$. Note that the degree shifts indicated in the complex above are with respect to the grading given by the $T_{i}$ 's, while the degree shifts with respect to the grading of $A$ are already contained in our definition of the $\mathcal{Z}_{i}$ 's. From now on, when we take the degree $\nu$ part of the approximation complex, denoted $\left(\mathcal{Z}_{\bullet}\right)_{\nu}$, it should always be understood to be taken with respect to the grading induced by $A$. Hereafter we denote by $\mathfrak{m}$ the ideal $\left(X_{1}, X_{2}, X_{3}, X_{4}\right) \subset A$.

\subsection{Acyclicity criterion}

Our first concern is to show that $\mathcal{Z}_{\bullet}\left(g_{1}, \ldots, g_{4} ; A\right)$, the approximation complex of cycles is acyclic under suitable assumptions. We have, similarly to [3, Lemma $2]$, the following 
Lemma 1 Suppose that $I=\left(g_{1}, g_{2}, g_{3}, g_{4}\right) \subset A$ is of codimension at least 2, and let $\mathscr{P}:=\operatorname{Proj}(A / I) \subset \mathscr{S}$. Then the following are equivalent:

(i) $\mathcal{Z}_{\bullet}$ is acyclic,

(ii) $\mathcal{Z}_{\bullet}$ is acyclic outside $V(\mathfrak{m})$,

(iii) $\mathscr{P}$ is locally defined by 3 equations (i.e. locally an almost complete intersection).

Proof. The proof is very similar to [3, Lemma 2]; the only difference is that $A$ is not a polynomial ring here, but it is still a Gorenstein ring which is the main required property for $A$. Observe that the lemma is unaffected by an extension of the base field, so one may assume that $\mathbb{K}$ is infinite.

By [11, Theorem 12.9], we know that $\mathcal{Z}_{\bullet}$ is acyclic (resp. acyclic outside $V(\mathfrak{m})$ ) if and only if $I$ is generated by a proper sequence (resp. $\mathscr{P}$ is locally defined by a proper sequence). Recall that a sequence $a_{1}, \ldots, a_{n}$ of elements in a commutative ring $B$ is a proper sequence if

$$
a_{i+1} H_{j}\left(a_{1}, \ldots, a_{i} ; B\right)=0 \text { for } i=0, \ldots, n-1 \text { and } j>0,
$$

where the $H_{j}$ 's denote the homology groups of the corresponding Koszul complex.

It is clear that (i) implies (ii). Assuming (ii), we will now deduce that $\mathscr{P}$ is locally defined by a proper sequence. As explained in [3, Lemma 2], one can choose $h_{1}, h_{2}, h_{3}, h_{4}$ to be sufficiently generic linear combinations of the $g_{i}$ 's such that

- $\left(h_{1}, \ldots, h_{4}\right)=\left(g_{1}, \ldots, g_{4}\right) \subset A$,

- $h_{1}, h_{2}$ is an $A$-regular sequence, which implies that $h_{1}, h_{2}, h_{3}$ is a proper sequence in $A$,

- $h_{1}, \ldots, h_{4}$ form a proper sequence outside $V(\mathfrak{m})$.

By [2, Theorem 1.6.16], we have

$$
H_{1}\left(h_{1}, h_{2}, h_{3} ; A\right) \simeq \operatorname{Ext}_{A}^{2}\left(A /\left(h_{1}, h_{2}, h_{3}\right), A\right)
$$

and since $A$ is Gorenstein (for it is a complete intersection), i.e. isomorphic to its canonical module [2, Theorem 3.3.7], then

$$
H_{1}\left(h_{1}, h_{2}, h_{3} ; A\right) \simeq \operatorname{Ext}_{A}^{2}(A / J, A) \simeq \omega_{A / J}
$$

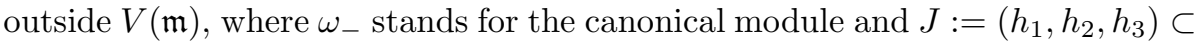
$A$. Since the annihilator of $\omega_{A / J}$ over $A$ is $\left(J: \mathfrak{m}^{\infty}\right) \subset A$ (observe that $A / J$ defines isolated points and use for instance [8, Corollary 21.3]), we deduce that $h_{4} \in\left(J: \mathfrak{m}^{\infty}\right)$, that is to say that $\mathscr{P}$ is locally defined by 3 equations.

Now, assume (iii). Similarly to what we did above, one can find $h_{1}, \ldots, h_{4}$ sufficiently generic linear combinations of the $g_{i}$ 's so that $h_{1}, h_{2}$ is an $A$-regular 
sequence and $h_{1}, h_{2}, h_{3}$ define $\mathscr{P}$. It follows that $h_{4} \in\left(J: \mathfrak{m}^{\infty}\right) \subset A$, where $J:=$ $\left(h_{1}, h_{2}, h_{3}\right) \subset A$, and hence (5) implies that $h_{4}$ annihilates $H_{1}\left(h_{1}, h_{2}, h_{3} ; A\right)$; it follows that $h_{1}, \ldots, h_{4}$ form a proper sequence in $A$, so $\mathcal{Z}_{\bullet}$ is acyclic.

As soon as the base points (if there are any) of the parametrization $\psi$ (or equivalently $\phi$ ) are isolated and locally defined by 3 equations, then its associated approximation complex of cycles is acyclic. Therefore, it can be used to compute and represent the codimension one part of the annihilator of the $A\left[T_{1}, \ldots, T_{4}\right]$-module $H^{0}\left(\mathcal{Z}_{\bullet}\right)$ which is nothing but the symmetric algebra $\operatorname{Sym}_{A}(I)$. Our interest in this module is motivated by the following

Lemma 2 Suppose that $\mathscr{P}:=\operatorname{Proj}(A / I)$ has dimension $\leq 0$ and is locally defined by 3 equations. If $\eta$ is an integer such that

$$
H_{\mathfrak{m}}^{0}\left(\operatorname{Sym}_{A}(I)\right)_{\nu}=0 \text { for all } \nu \geq \eta,
$$

then, for all $\nu \geq \eta$ we have

$$
\operatorname{ann}_{\mathbb{K}[\underline{T}]}\left(\operatorname{Sym}_{A}(I)_{\nu}\right)=\operatorname{ann}_{\mathbb{K}[\underline{T}]}\left(\operatorname{Sym}_{A}(I)_{\eta}\right) \subseteq \operatorname{ker}(h) .
$$

Moreover, the above inclusion is an equality if $\mathscr{P}$ is locally defined by 2 equations.

Proof. For all $\nu \geq \eta$, the equality

$$
\operatorname{ann}_{\mathbb{K}[\underline{T}]}\left(\operatorname{Sym}_{A}(I)_{\nu}\right)=\operatorname{ann}_{\mathbb{K}[T]}\left(\operatorname{Sym}_{A}(I)_{\eta}\right)
$$

is proven in [5, Proposition 5.1] for $A=\mathbb{K}[\underline{X}]$. However, the same proof can be applied without modifications to our setting: The key property used in the proof is the fact that the canonical map $A_{1} \otimes A_{n} \rightarrow A_{n+1}$ is surjective and this is also valid for $A=\mathbb{K}[\underline{X}] /\left(X_{1} X_{4}-X_{2} X_{3}\right)$. Moreover, by (任) we have that $\operatorname{ann}_{\mathbb{K}[T]}\left(\operatorname{Sym}_{A}(I)_{\nu}\right) \neq 0$ for $\nu \gg 0$ if and only if $\mathscr{P}$ is locally generated by at most 3 equations, and in this case it is clear that it is contained in $\operatorname{ker}(h)$. Finally, if $\mathscr{P}$ is locally defined by at most 2 equations, meaning that $\mathscr{P}$ is locally a complete intersection, then $I$ is of linear type outside $V(\mathfrak{m})$ (use for instance [5. Propositions 4.1 and 4.5]) which shows the last claimed equality as proven in [5, Proposition 5.1].

In other words, if the base points of the parametrization are isolated and locally complete intersections then certain graded parts of the approximation complex $\mathcal{Z}_{\bullet}$ yield a way to compute an implicit equation of $\mathscr{H}$. Our next task is to explicitly describe the saturation index of the symmetric algebra, i.e. the integer $\eta$ appearing in Lemma 2. This will provide us with the key tool for developing the algorithm presented in Section 1 .

\subsection{The saturation index}

For any ideal $J$ of $A$ we denote by $J^{\text {sat }}$ the saturation of $J$ with respect to the ideal $\mathfrak{m}$, i.e. $J^{\text {sat }}:=\left(J:_{A} \mathfrak{m}^{\infty}\right) \subset A$. Also, we recall that if $M$ is a $\mathbb{N}$-graded 
$B$-module, where $B$ is a $\mathbb{N}$-graded ring, its initial degree is defined as

$$
\operatorname{indeg}(M):=\min \left\{\nu \in \mathbb{N}: M_{\nu} \neq 0\right\} \geq 0 .
$$

With these notations, we have

Theorem 1 If $\mathscr{P}:=\operatorname{Proj}(A / I)$ is a zero-dimensional scheme (i.e. supported on a finite number of points, possibly zero) then

$$
H_{\mathfrak{m}}^{0}\left(\operatorname{Sym}_{A}(I)\right)_{\nu}=0 \quad \forall \nu \geq 2 d-1-\operatorname{indeg}\left(I^{\mathrm{sat}}\right) .
$$

The proof of this theorem is actually similar to the proof of 3, Theorem 4]. The difference is that in our case the $\operatorname{ring} A$ is not a polynomial ring but a quotient ring. So to validate the proof of [3, Theorem 4] we have to make explicit the local cohomology and the dualizing module of $A$ which is, as a complete intersection, a Gorenstein ring (the key property for what follows). We state these results in a little more general case for the sake of clarity.

Proposition 2 Let $k$ be a commutative Noetherian ring and $C:=k\left[X_{1}, \ldots, X_{n}\right]$, with $n \geq 1$, which is canonically graded by $\operatorname{deg}\left(X_{i}\right)=1$ for all $i=1, \ldots, n$. Suppose given a homogeneous polynomial $f$ of degree $r \geq 1$ and consider the graded quotient ring $B:=C /(f)$. The following properties hold:

- $\omega_{B} \simeq B(-n+r)$, a graded isomorphism where $\omega_{B}$ stands for the canonical module of $B$,

- $H_{\mathfrak{m}}^{i}(B)=0$ if $i \neq n-1$ and for all $\nu \in \mathbb{Z}$

$$
H_{\mathfrak{m}}^{n-1}(B)_{\nu} \simeq B(-n+r)_{-\nu}
$$

- if $K \bullet$ denotes the Koszul complex associated to a given sequence $\left(a_{1}, \ldots, a_{s}\right)$ of homogeneous elements in $B$ of degree $d_{1}, \ldots, d_{s}$ respectively, then we have the isomorphisms

$$
H_{\mathfrak{m}}^{n-1}\left(K_{\bullet}\right)_{\nu} \simeq \operatorname{Hom}_{B / \mathfrak{m}}\left(K_{s-\bullet}\left(\sum_{i=1}^{s} d_{i}-n+r\right)_{-\nu}, B / \mathfrak{m}\right) .
$$

Proof. To prove the first claim, we first recall that we have $\omega_{C} \simeq C(-n)$. Then, [2. Corollary 3.6.14] shows that

$$
\omega_{B} \simeq\left(\omega_{C} / f \cdot \omega_{C}\right)(r) \simeq B(-n+r) .
$$

For the second claim, we recall that the local cohomology of $C$ is well-known: $H_{\mathfrak{m}}^{i}(C)=0$ for all $i \neq n$ and

$$
H_{\mathfrak{m}}^{n}(C)_{\nu} \simeq C_{-n-\nu}
$$

for all $\nu \in \mathbb{Z}$. Now, the exact sequence

$$
0 \rightarrow C(-r) \stackrel{\times f}{\rightarrow} C \rightarrow B \rightarrow 0
$$


whose long exact cohomology sequence contains the segments

$$
H_{\mathfrak{m}}^{j}(C) \rightarrow H_{\mathfrak{m}}^{j}(B) \rightarrow H_{\mathfrak{m}}^{j+1}(C(-r))
$$

implies that $H_{\mathfrak{m}}^{j}(B)=0$ for all $j<n-1$ as for $j+1<n$ both the left and the right hand side vanish. Furthermore, the segment

$$
0 \rightarrow H_{\mathfrak{m}}^{n-1}(B) \rightarrow H_{\mathfrak{m}}^{n}(C(-r)) \rightarrow H_{\mathfrak{m}}^{n}(C)
$$

taken in degree $\nu$ shows

$$
H_{\mathfrak{m}}^{n-1}(B)_{\nu}=\operatorname{ker}\left(H_{\mathfrak{m}}^{n}(C(-r))_{\nu} \rightarrow H_{\mathfrak{m}}^{n}(C)_{\nu}\right) .
$$

By the self-duality of the Koszul complex and (6) this later equals exactly $B_{-\nu-n+r}$. Finally, since $\operatorname{dim}(C)=n$ we have $\operatorname{dim}(B)=n-1$ which implies that $H_{\mathfrak{m}}^{j}(B)=0$ for $j>n-1$ by [2, Theorem 3.5.7].

The third claim is a direct generalization of the classical property

$$
H_{\mathfrak{m}}^{n}\left(K_{\bullet}\right)_{\nu} \simeq \operatorname{Hom}_{C / \mathfrak{m}}\left(K_{s-\bullet}\left(\sum_{i=1}^{s} d_{i}-n\right)_{-\nu}, C / \mathfrak{m}\right) .
$$

The only thing which changes is the shift by $r$ in the canonical module of $B$ and the dimension of $B$ which is $n-1$ whereas $\operatorname{dim}(C)=n$.

Proof of theOREM 1. We consider the two spectral sequences associated to the double complex $H_{\mathfrak{m}}^{\circ}\left(\mathcal{Z}_{\bullet}\right)$ :

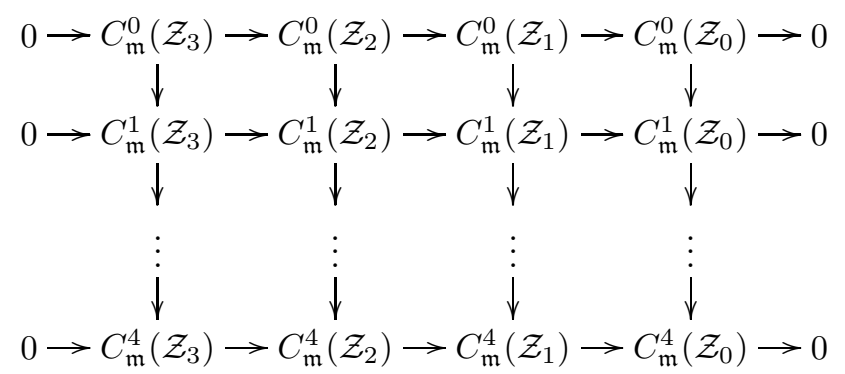

They both converge to the hypercohomology of $\mathcal{Z}_{\boldsymbol{\bullet}}$. One of them stabilizes at level two with:

$$
{ }_{2}^{\prime} E_{q}^{p}={ }_{\infty}{ }^{\prime} E_{q}^{p}=\left\{\begin{array}{cl}
H_{\mathfrak{m}}^{p}\left(H_{q}\left(\mathcal{Z}_{\bullet}\right)\right) & \text { for } p=0,1 \text { and } q>0 \\
H_{\mathfrak{m}}^{p}\left(\operatorname{Sym}_{A}(I)\right) & \text { for } q=0 \\
0 & \text { else. }
\end{array}\right.
$$

and the other one gives at level one:

$$
{ }_{1}^{\prime \prime} E_{q}^{p}=H_{\mathfrak{m}}^{p}\left(Z_{q}\right)[q d] \otimes_{A} A[\underline{T}](-q) .
$$


As explained in [3, Theorem 4], the comparison of these two spectral sequences and [3, Lemma 1] show ${ }^{2}$ that the module $H_{\mathfrak{m}}^{0}\left(\operatorname{Sym}_{A}(I)\right)_{\nu}$ vanishes as soon as $\left({ }_{1}{ }^{\prime \prime} E_{p}^{p}\right)_{\nu}$ vanishes for $p=2,3$. Moreover, setting $-^{\star}:=\operatorname{Homgr}_{A}(-, A / \mathfrak{m})$, we have the graded isomorphisms

$$
{ }_{1}^{\prime \prime} E_{3}^{3} \simeq(A / I)^{\star}[2-d] \otimes_{A} A[\underline{T}](-3)
$$

and

$$
1^{\prime \prime} E_{2}^{2} \simeq\left(I^{\text {sat }} / I\right)^{\star}[2-2 d] \otimes A[\underline{T}](-2) .
$$

It follows that $\left({ }_{1}{ }^{\prime \prime} E_{2}^{2}\right)_{\nu}$ and $\left({ }_{1}{ }^{\prime \prime} E_{3}^{3}\right)_{\nu}$ vanish simultaneously if

$$
\nu>\min \left(d-2,2 d-2-\operatorname{indeg}\left(I^{\mathrm{sat}} / I\right)\right) .
$$

This is true whenever $\nu \geq \nu_{0}:=2 d-1-\operatorname{indeg}\left(I^{\text {sat }}\right)$, $\operatorname{since} \min \left(d, \operatorname{indeg}\left(I^{\text {sat }} / I\right)\right)=$ $\operatorname{indeg}\left(I^{\text {sat }}\right)$.

Remark 1 Since $I$ is generated in degree $d$ and $I \subset I^{\text {sat }}$ we have the inequality $0 \leq \operatorname{indeg}\left(I^{\text {sat }}\right) \leq d$. It follows that

$$
d-1 \leq 2 d-1-\operatorname{indeg}\left(I^{\text {sat }}\right) \leq 2 d-1 .
$$

The lower bound is reached whenever the ideal $I$ is saturated (meaning $I=$ $I^{\text {sat})}$ and the higher bound corresponds to the abscence of base points of the parametrization.

\subsection{The main result}

We now have all the tools necessary at our disposal and can proceed to the main result of this paper. But before, recall that there are two distinct notions of multiplicity for a base point $\mathfrak{p} \in V(I) \cap \mathscr{S} \subset \mathbb{P}^{3}$ : the algebraic multiplicity denoted $e_{\mathfrak{p}}$ and the geometric multiplicity denoted $d_{\mathfrak{p}}$ (see for instance [5, $\left.\$ 2.2\right]$ for more details).

Theorem 2 Assume that $\operatorname{dim} \mathscr{P}:=\operatorname{Proj}(A / I) \leq 0$ and that $\mathscr{P}$ is locally an almost complete intersection (i.e. locally defined by 3 equations). Then, for every integer

$$
\nu \geq \nu_{0}:=2 d-1-\operatorname{indeg}\left(I^{\text {sat }}\right)
$$

the determinant $D$ of the complex $\left(\mathcal{Z}_{\bullet}\right)_{\nu}$ of $\mathbb{K}[\underline{T}]$-modules (which is unique up to multiplication by a non-zero constant in $\mathbb{K}$ ) is a non-zero homogeneous element in $\mathbb{K}[\underline{T}]$, independent of $\nu \geq \nu_{0}$ and of degree

$$
2 d^{2}-\sum_{\mathfrak{p} \in V(I) \cap \mathscr{S} \subset \mathbb{P}^{3}} d_{\mathfrak{p}}
$$

such that $D=F^{\operatorname{deg}(\psi)} G$ where $F$ is the implicit equation of $\mathscr{H}, G$ is coprime with $F$ and $\operatorname{deg}(G)=\sum_{\mathfrak{p} \in V(I) \cap \mathscr{S}}\left(e_{\mathfrak{p}}-d_{\mathfrak{p}}\right)$.

Moreover, $G \in \mathbb{K} \backslash\{0\}$ if and only if $\mathscr{P}$ is locally a complete intersection (i.e. locally defined by 2 equations).

\footnotetext{
${ }^{2}$ Note that [3, Lemma 1] can be applied verbatim in our case (modulo some little change on the degree shifts that we will describe below) because of Proposition 2 .
} 
Proof. First of all, observe that $D$ is independent of $\nu$ by THEOREM 1 . It is an homogeneous element of $\mathbb{K}[\underline{T}]$ because $\left(\mathcal{Z}_{\bullet}\right)_{\nu}$ is a graded complex of $\mathbb{K}[\underline{T}]$ modules and it is non-zero because $\mathscr{P}$ is locally an almost complete intersection, a fact we already used in LEMMA 2 .

The computation of $\operatorname{deg}(D)$ can be done as in [3, Theorem 4]: For $\nu \gg 0$ we have

$$
\operatorname{deg}(D)=\operatorname{dim}\left(Z_{1}\right)_{\nu+d}-2 \operatorname{dim}\left(Z_{2}\right)_{\nu+2 d}+3 \operatorname{dim}\left(Z_{3}\right)_{\nu+3 d} .
$$

In the case where all the $H_{i}$ 's, with $i>0$, vanish then $\operatorname{deg}(D)=2 d^{2}$. If $H_{1}$ and $H_{2}$ are non-zero, then they contribute to the above quantity for

$$
\begin{aligned}
\operatorname{dim}\left(H_{1}\right)_{\nu+d}-\operatorname{dim}\left(H_{2}\right)_{\nu+d}- & 2 \operatorname{dim}\left(H_{2}\right)_{\nu+2 d} \\
& =\operatorname{dim}\left(H_{0}\right)_{\nu+d}-2 \operatorname{dim}\left(H_{2}\right)_{\nu+2 d}=-\operatorname{deg} \mathscr{P}
\end{aligned}
$$

where we assume that $\nu \gg 0$, since $H_{2} \simeq \omega_{A / I}$. Therefore, we deduce that

$$
\operatorname{deg}(D)=2 d^{2}-\operatorname{deg} \mathscr{P}=2 d^{2}-\sum_{\mathfrak{p} \in V(I) \cap \mathscr{S} \subset \mathbb{P}^{3}} d_{\mathfrak{p}}
$$

Now, setting $\mathfrak{q}:=\operatorname{ker}(h)$ and using standard properties of determinants of complexes we compute

$$
\begin{aligned}
{\left[\operatorname{det}\left(\left(\mathcal{Z}_{\bullet}\right)_{\nu}\right)\right] } & =\operatorname{div}\left(H_{0}\left(\mathcal{Z}_{\bullet}\right)\right) \\
& =\operatorname{div}\left(\operatorname{Sym}_{A}(I)_{\nu}\right) \\
& =\sum_{\mathfrak{p} \operatorname{prime}, \operatorname{codim}(\mathfrak{p})=1} \operatorname{length}\left(\left(\operatorname{Sym}_{A}(I)_{\nu}\right)_{\mathfrak{p}}\right) \cdot[\mathfrak{p}] \\
& =\operatorname{length}\left(\left(\operatorname{Sym}_{A}(I)_{\nu}\right)_{\mathfrak{q}}\right) \cdot[\mathfrak{q}]+\cdots
\end{aligned}
$$

Since length $\left(\left(\operatorname{Sym}_{A}(I)_{\nu}\right)_{\mathfrak{q}}\right)=\operatorname{deg}(\psi)$ as proved in [5, Theorem 5.2], we deduce that $D=F^{\operatorname{deg}(\psi)} G$ where $G$ does not divide $F$.

Finally, using equations (3) and (8) we deduce that

$$
\operatorname{deg}(G)=\sum_{\mathfrak{p} \in V(I) \cap \mathscr{S}}\left(e_{\mathfrak{p}}-d_{\mathfrak{p}}\right),
$$

and it is well-known that $e_{\mathfrak{p}} \geq d_{\mathfrak{p}}$ with equality if and only if the point $\mathfrak{p}$ is locally a complete intersection.

Recall that the determinant of the complex $\left(\mathcal{Z}_{\bullet}\right)_{\nu}$ can either be obtained as an alternating product over some sub-determinants of the matrices appearing in the complex or as a $g c d$ of maximal minors of the first map in the $\left(\mathcal{Z}_{\bullet}\right)_{\nu}$-complex (we will explicitly construct this matrix $M$ in the next section). One can either compute this gcd directly or factorize one of the maximal minors, however, both methods are computationally expensive (as all existing implicitization methods). 
From a practical point of view, it might be interesting to avoid the actual computation of an implicit equation and use instead the matrix $M$ as an implicit representation of the surface, since it is more compact and much easier to compute. To give an example, let us suppose that we are in the case of locally complete intersection base points. Then if we want to decide if a given point $P$ lies on the surface there is no need to compute the implicit equation: It suffices to evaluate $M$ in this point, as the rank of $M$ drops if and only if $P$ belongs to the surface.

This is due to the fact that for a commutative ring $R$ and a morphism $\alpha: R^{m} \rightarrow R^{n}$ with $m \geq n$ we always have

$$
\operatorname{ann}_{R}(\operatorname{coker}(\alpha))^{n} \subseteq I_{n}(\alpha) \subseteq \operatorname{ann}_{R}(\operatorname{coker}(\alpha))
$$

where $I_{n}(\alpha)$ denotes the ideal generated by the maximal minors of the matrix of $\alpha$, i.e. the principal Fitting ideal of $\alpha$ (see for instance [8, Proposition 20.7]). Ours is the special case $R=\mathbb{K}[\underline{T}]$ and $\alpha$ is the first map in $\left(\mathcal{Z}_{\bullet}\right)_{\nu}$, i.e. the one induced by $e_{1}$, and hence $\operatorname{coker}(\alpha)=\operatorname{Sym}_{A}(I)_{\nu}$. Geometrically, this means that the maximal minors of $M$ define the hypersurface $\mathscr{H}$ by LEMMA 2 , and consequently, the points for which the rank of $M$ drops are exactly those belonging to $\mathscr{H}$.

Similarly, other problems arising from applications might be solved by direct computations using the matrix representation without the (expensive) transition to the implicit equation.

\section{Algorithm}

In order to show explicitly how the theoretical results from the previous sections are used in practice, we formulate an algorithm for the actual computation of the matrix representing the implicit equation. It is efficient and easy to implement, as it consists basically of the resolution of a linear system. We give only the essential steps, see [3, Section 3] for a more detailed description of a very similar algorithm.

- Given four bi-homogeneous polynomials $f_{1}, f_{2}, f_{3}, f_{4}$ of degree $d$, define the homogeneous polynomials $g_{1}, g_{2}, g_{3}, g_{4} \in A=\mathbb{K}[\underline{X}] /\left(X_{1} X_{4}-X_{2} X_{3}\right)$ of the same degree by setting $g_{i}=\omega\left(f_{i}\right)$, where $\omega$ is the isomorphism defined in Section 2 .

- Find the solution space $W$ of the linear system (over $\mathbb{K}$ ) defined by

$$
\sum_{i \in\{1, \ldots, 4\}} a_{i} g_{i}=0
$$

where $\left(a_{1}, a_{2}, a_{3}, a_{4}\right) \in\left(A_{\nu_{0}}\right)^{4}$ and $\nu_{0}=2 d-1-\operatorname{indeg}\left(I^{\text {sat }}\right)$, i.e. one writes the equation with respect to a basis of $A_{\nu_{0}+d}$ and compares the coefficients. $W$ is represented by a $\operatorname{dim}_{\mathbb{K}}\left(A_{\nu_{0}+d}\right) \times 4 \operatorname{dim}_{\mathbb{K}}\left(A_{\nu_{0}}\right)$-matrix $N$, where the first $k:=\operatorname{dim}_{\mathbb{K}}\left(A_{\nu_{0}}\right)$ columns represent the coefficients of $a_{1}$, the next $k$ coefficients $a_{2}$, etc. 
- For $i \in\{1, \ldots, 4\}$, let $M_{i}$ be the $k \times k$-matrix $T_{i} \cdot \operatorname{Id}_{k}$. Then

$$
M:=N \cdot\left(\begin{array}{c}
M_{1} \\
\vdots \\
M_{4}
\end{array}\right)
$$

is a matrix of the first map of the graded part $\left(\mathcal{Z}_{\bullet}\right)_{\nu_{0}}$ of the approximation complex.

As we proved, in the case where the base points of the parametrization $\phi$ are isolated and locally complete intersections, $M$ represents the surface $\mathscr{H}$. Also, the $g c d$ of the maximal minors (of size $k$ ) of $M$ equals its implicit equation.

\section{An illustrative example}

We now present an example to illustrate our method, which provides a matrixbased representation of the implicit equation of $\mathscr{H}$ by means of the linear syzygies of its parametrization $\phi$ (or more precisely, of $\psi$ ). It should be emphasized that all the following computations are presented in order to explore in detail our approach and are not all required to get the expected matrix-based representation. Our code is written for Macaulay2 (see 10]), in which one can easily compute all the terms and maps of the approximation complex.

Consider the following example taken from [1, Example 4.16]:

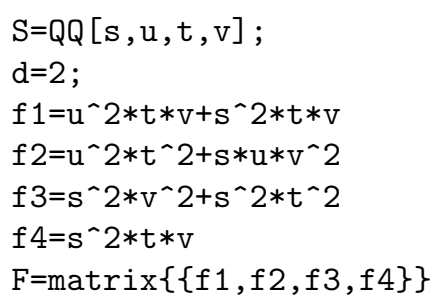

Note that the interested reader can experiment with his own example just by changing the above definitions of the polynomials $f_{1}, f_{2}, f_{3}, f_{4}$ giving the parametrization.

The first thing to do is to use the isomorphism $\bar{\theta}$ to switch from $S$ (note that the ring $S$ defined in the above command is not exactly the ring $S$ we have introduced in Section 2) to the $\operatorname{ring} A$ :

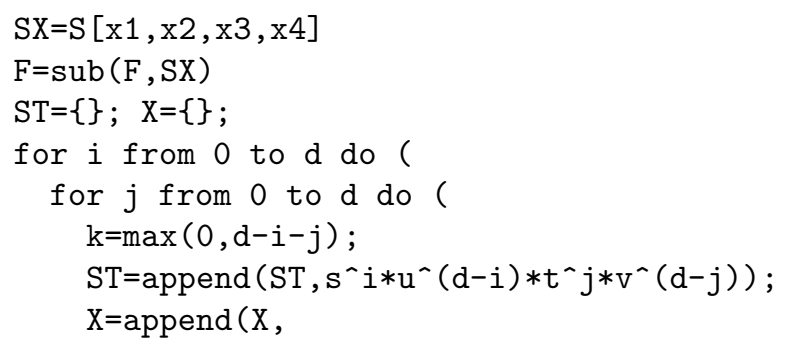




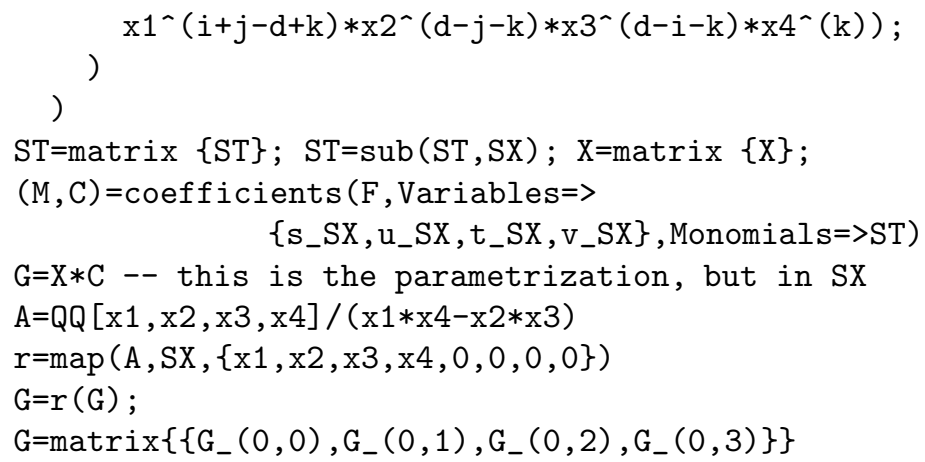

The matrix $G$ is the matrix (with entries in $A$ ) of the parametrization $\psi$ from the Segre variety $\mathscr{S}$ to $\mathbb{P}^{3}$. One should note that the quotient ring $A$ is a very simple quotient ring: essentially, computations in $A$ can be done in $\mathbb{K}\left[X_{1}, \ldots, X_{4}\right]$ modulo the substitution of $X_{1} X_{4}$ by $X_{2} X_{3}$. Moreover, bases for $A$ in any given degrees can easily be pre-computed since they do not depend on the given parametrization $\phi$.

We can now define the terms of the approximation complex of cycles $\mathcal{Z}_{\bullet}$ :

$\mathrm{Z} 0=\mathrm{A}^{\wedge} 1$

Z1=kernel $\operatorname{koszul}(1, G)$;

$\mathrm{Z} 2=\operatorname{kernel} \operatorname{koszul}(2, \mathrm{G})$;

Z3=kernel $\operatorname{koszul}(3, G)$;

As we already remarked, $\mathcal{Z}_{4}=0$. Define the integer

$n u=2 * d-1$

We can compute the Euler characteristic of $\left(\mathcal{Z}_{\bullet}\right)_{\nu}$ and check that it is zero with the command

hilbertFunction (nu, Z0)-hilbertFunction (nu+d, Z1) +

hilbertFunction $(n u+2 * d, Z 2)$-hilbertFunction $(n u+3 * d, Z 3)$

and also compute the degree of $D$, the determinant of the complex $\left(\mathcal{Z}_{\bullet}\right)_{\nu}$, with the command

hilbertFunction (nu+d, Z1) $-2 *$ hilbertFunction $(n u+2 * d, Z 2)$

$+3 *$ hilbertFunction $(n u+3 * d, Z 3)$

This number equals the degree of $\mathscr{H}$ if all the base points, if any, form locally a complete intersection. In this example, we find degree 7.

At this step, one can try to lower the integer $\nu$ according to THEOREM 2; to this end we compute the degrees of the generators of the saturation of the ideal $\left(g_{1}, \ldots, g_{4}\right)$ :

degrees gens saturate(ideal $\mathrm{G}$, ideal $(\mathrm{x} 1, \mathrm{x} 2, \mathrm{x} 3, \mathrm{x} 4)$ ) 
Since, in this example, the smallest degree is 1 we can redefine

$\mathrm{nu}=2 * \mathrm{~d}-2$

and we can re-check the Euler characteristic and the degree of the determinant of $\left(\mathcal{Z}_{\bullet}\right)_{\nu}$.

We can now compute the matrix of the first map of $\left(\mathcal{Z}_{\bullet}\right)_{\nu}$, that is to say the matrix of linear syzygies of $g_{1}, \ldots, g_{4}$ which represents $\mathscr{H}$ :

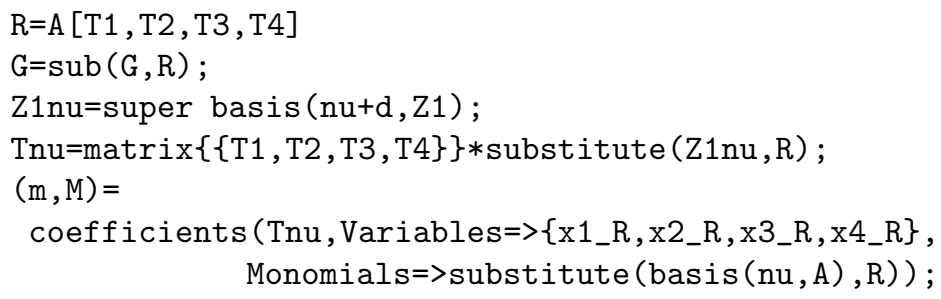

The matrix $\mathrm{M}$ is the desired matrix, and it is of size $9 \times 12$.

\section{Comments and conclusion}

We have presented a new approach to compute an implicit representation in terms of linear syzygies for a surface in $\mathbb{P}^{3}$ parametrized by bi-homogeneous polynomials of bi-degree $(d, d), d \geq 1$, under the assumption that the base points are isolated and locally complete intersections. This result, along with the similar ones for parametrizations over the projective plane, shows that in many cases it is not necessary to use quadratic syzygies in order to represent the implicit equation of a surface.

We should point out that this method has the advantages of being valid in a very general setting (we have neither assumed birationality nor made other additional assumptions on the parametrization) and of working well in the presence of base points. Furthermore, the matrix representing the surface can be computed in a very efficient way.

It would be nice if we could use the same method for mixed degrees as well, i.e. consider parametrizations by bi-homogeneous polynomials of bi-degree $\left(d_{1}, d_{2}\right)$ with $d_{1}, d_{2} \geq 1$. Let us discuss some ideas on how to generalize to the mixed case:

- Putting weights on the variables in $S$ will not give us good properties for $S$, for instance $S$ will not be generated by $S_{1}$ as an $S_{0}$ algebra in general.

- Considering the bi-degree $\left(\max \left(d_{1}, d_{2}\right), \max \left(d_{1}, d_{2}\right)\right)$ is not possible because it introduces a base point locus of positive dimension and we will lose the acyclicity of the approximation complex.

- One way to come back to unmixed bi-degree is to make the substitutions

$$
s \leftarrow s^{\operatorname{lcm}\left(d_{1}, d_{2}\right) / d_{1}} \text { and } t \leftarrow t^{\operatorname{lcm}\left(d_{1}, d_{2}\right) / d_{2}} .
$$


Everything works fine in this case, but we are not representing $F^{\operatorname{deg}(\psi)}$, but $F^{\operatorname{deg}(\psi)} \operatorname{lcm}\left(d_{1}, d_{2}\right) / \operatorname{gcd}\left(d_{1}, d_{2}\right)$ which is is not optimal, as it increases the size of the matrices involved. For instance, we could treat Example 10 from [12] in this way. It is a surface of bi-degree $(2,3)$ defined by

$$
\begin{aligned}
& f_{1}=\left(t+t^{2}\right)(s-1)^{2}+\left(1+s t-s^{2} t\right)(t-1)^{2} \\
& f_{2}=\left(-t-t^{2}\right)(s-1)^{2}+\left(-1+s t+s^{2} t\right)(t-1)^{2} \\
& f_{3}=\left(t-t^{2}\right)(s-1)^{2}+\left(-1-s t+s^{2} t\right)(t-1)^{2} \\
& f_{4}=\left(t+t^{2}\right)(s-1)^{2}+\left(-1-s t-s^{2} t\right)(t-1)^{2}
\end{aligned}
$$

By replacing $s$ by $s^{3}$ and $t$ by $t^{2}$, we obtain a parametrization of bi-degree $(6,6)$ and $F^{6}$ can indeed be computed in degree $\nu \geq 2 \cdot 6-1-6=5$ of the approximation complex as the $g c d$ of the maximal minors of a $42 \times 36$ matrix, whereas in the original paper it was computed as the determinant of a $5 \times 5$-matrix.

Therefore, it seems that the tools we used above (and which work well for unmixed bi-degree) are not well-suited for this more general case and that it might be necessary to take into account the bi-graded structure of $\mathrm{S}$ in order to devise a method that is adapted to mixed bi-degrees. We hope to develop this in the near future.

\section{Acknowledgments}

The authors have been partially supported by the French ANR "Gecko".

\section{References}

[1] W. A. Adkins, J. W. Hoffman, and H. H. Wang. Equations of parametric surfaces with base points via syzygies. J. Symbolic Comput., 39(1):73-101, 2005 .

[2] W. Bruns and J. Herzog. Cohen-Macaulay rings, volume 39 of Cambridge Studies in Advanced Mathematics. First edition. Cambridge University Press, Cambridge, 1993.

[3] L. Busé and M. Chardin. Implicitizing rational hypersurfaces using approximation complexes. J. Symbolic Comput., 40(4-5):1150-1168, 2005.

[4] L. Busé, D. Cox, and C. D'Andrea. Implicitization of surfaces in $\mathbb{P}^{3}$ in the presence of base points. J. Algebra Appl., 2(2):189-214, 2003.

[5] L. Busé and J.-P. Jouanolou. On the closed image of a rational map and the implicitization problem. J. Algebra, 265(1):312-357, 2003. 
[6] D. Cox. Curves, surfaces, and syzygies. In Topics in algebraic geometry and geometric modeling, volume 334 of Contemp. Math., pages 131-150. Amer. Math. Soc., Providence, RI, 2003.

[7] D. A. Cox. Equations of parametric curves and surfaces via syzygies. In Symbolic computation: solving equations in algebra, geometry, and engineering (South Hadley, MA, 2000), volume 286 of Contemp. Math., pages 1-20. Amer. Math. Soc., Providence, RI, 2001.

[8] D. Eisenbud. Commutative algebra, volume 150 of Graduate Texts in Mathematics. Springer-Verlag, New York, 1995.

[9] W. Fulton. Intersection theory, volume 2 of Ergebnisse der Mathematik und ihrer Grenzgebiete (3) [Results in Mathematics and Related Areas (3)]. Springer-Verlag, Berlin, 1984.

[10] D. R. Grayson and M. E. Stillman. Macaulay 2, a software system for research in algebraic geometry. Available at http://www.math.uiuc.edu/Macaulay2/.

[11] J. Herzog, A. Simis, and W. V. Vasconcelos. Koszul homology and blowingup rings. In Commutative algebra (Trento, 1981), volume 84 of Lecture Notes in Pure and Appl. Math., pages 79-169. Dekker, New York, 1983.

[12] A. Khetan and C. D'Andrea. Implicitization of rational surfaces using toric varieties. J. Algebra, 303(2):543-565, 2006.

[13] T. Sederberg and F. Chen. Implicitization using moving curves and surfaces. Computer Graphics Annual Conference Series, pages 301-308, 1995.

[14] W. V. Vasconcelos. Arithmetic of blowup algebras, volume 195 of London Mathematical Society Lecture Note Series. Cambridge University Press, Cambridge, 1994. 